\title{
Author Correction: Towards individualized therapy for metastatic renal cell carcinoma
}

Ritesh R. Kotecha (i), Robert J. Motzer(i) and Martin H. Voss(i)

Nature Reviews Clinical Oncology (2019) https://doi.org/10.1038/s41571-019-0209-1

Published online 16 April 2019

The competing interests section of the HTML and PDF versions of this manuscript originally did not include the research support received by Robert J. Motzer from Eisai. This information has been added to the competing interests section of the HTML and PDF versions of the manuscript.

https://doi.org/10.1038/s41571-019-0214-4 I Published online 25 April 2019 